Tampa, Florida USA • December 1998

\title{
Design of switching controllers for systems with changing dynamics
}

\author{
Miloš Žefran and Joel W. Burdick \\ Department of Mechanical Engineering, MC 104-44 \\ California Institute of Technology, Pasadena, CA 91125 \\ $\{$ milos, jwb\}@robby.caltech.edu
}

\begin{abstract}
We present a framework for designing stable control schemes for systems with changing dynamics (SCD). Such systems form a subset of hybrid systems; their stabilization is therefore a problem in hybrid control. It is often difficult or even impossible to design a single controller that would stabilize a SCD. An appealing alternative are switching control schemes, where a different controller is employed in each dynamic regime and the stability of the overall system is ensured through an appropriate switching scheme. We formulate a set of sufficient conditions for the stability of a switching control scheme. We show that by imposing a hierarchy among the controllers, sufficient conditions can be formulated in a form suitable for the controller design. The hierarchy is formally defined through a partial order. Our methodology is applied to stabilization of a two-wheel mobile robot of the Hilare type, where the wheels are allowed to slip.
\end{abstract}

Keywords: systems with changing dynamics, hybrid systems, switching controllers, partial order, Hilare robot

\section{Introduction}

Design of controllers for hybrid systems remains a challenging problem and is the subject of considerable research. A common assumption of many existing design methodologies is that a physical plant with continuous dynamics is controlled by a supervisor in the form of a finite automaton. Our work addresses a different problem. We study systems whose dynamics change in different regions of the state space. Because of the inherently discontinuous dynamics, the control task for such systems becomes in many respects more challenging. In this paper, we study stabilization of systems with changing dynamics (SCD). The goal of control is to bring the system into a desired dynamic regime and stabilize an equilibrium set in that regime. Because of discontinuous dynamics, it is difficult to design a single controller that would stabilize a SCD. To reduce the complexity of the problem we propose an alternative strategy, whereby a different controller is employed in each dynamic regime; as the system evolves, we switch among different controllers. One of the goals of this paper is to investigate what conditions must be satisfied by such a switching controller to guarantee the stability of the system. A second goal is to suggest a paradigm for designing controllers that satisfy such conditions. We observe that in many tasks the stability in the sense of Lyapunov is too restrictive, so we concentrate on the control schemes that only guarantee the convergence of the trajectories to the desired set. However, our basic methodology does not preclude Lyapunov stability.

Majority of works on hybrid controller design rely on certain properties of the system. An early work is [1], where piecewise-linear systems are used as underlying model for hybrid systems. Algorithms for automated design of controllers for a simplified version of hybrid automata are described in [2]. A game-theoretic framework for design of hybrid controllers was proposed in [3]. In [4], timed Petri nets are used to model hybrid systems; supervisory control framework is employed for their control. A hybrid controller for so called cascade systems was proposed in [5].

A number of authors considered stability of hybrid systems. Classical Lyapunov theory was extended for non-smooth and hybrid systems in [6, 7]. Multiple Lyapunov functions were proposed for stability analysis of hybrid systems in $[8,9,10]$. A controller design methodology based on multiple Lyapunov functions is described in [11]. An important contribution towards the application of multiple Lyapunov functions for practical controller design is the work in [12] and [13]. The idea of guiding the system through a sequence of equilibrium points in order to stabilize it was employed in [14]. An assumption that is common to most of these works (an exception is [14]) is that every subsystem has the same equilibrium point which has to be stabilized. However, hybrid systems can exhibit much richer behavior: the system might switch between multiple equilibrium sets before reaching the final state. It is also commonly assumed that the switches between the controllers are either explicitly controlled, or that the switching surfaces can be explicitly characterized. These assumptions are quite restrictive and one of our goals is to relax them.

\section{Modeling}

Definition 2.1 $A$ system with changing dynamics (SCD) is a tuple $S C D=(\Xi, \mathcal{M}, \mathcal{U}, \Gamma, \mathcal{F}, \Sigma)$ where

1. $\Xi \subset \mathbb{Z}$ is a (finite) set of discrete states.

2. $\mathcal{M}=\left\{M_{i}\right\}_{i \in \Xi}$ is a collection of (differentiable, connected) manifolds. For simplicity, we assume $M_{i} \subseteq$ 
$\mathbb{R}^{n}$ for some $n$

3. $\mathcal{U} \subset \mathbb{R}^{m}$ is the set of continuous inputs.

4. $\Gamma \subset \mathbb{Z}$ is the set of discrete inputs.

5. $\mathcal{F}=\left\{f_{i}: M_{i} \times \mathcal{U} \rightarrow T M_{i}\right\}_{i \in \Xi}$ is a set of vector fields.

6. $\Sigma: \Xi \times \cup_{i \in \Xi} M_{i} \times \Gamma \times \mathcal{U} \rightarrow \Xi$ is a function describing the discrete evolution of the system.

The collection of manifolds $\mathcal{M}$ reflects the changing dynamics. On each manifold $M_{i}$, the system is described with a set of equations:

$$
\dot{x}_{i}=f_{i}(x, u),
$$

where $x \in M_{i}$ and $u \in u$. The system evolves on $M_{i}$ following the vector field $f_{i}$ as long as $\Sigma(i, x, \eta, u)=i$. When $\Sigma(i, x, \eta, u)$ becomes equal to $j \neq i$, the system dynamics switches to $\left(M_{j}, f_{j}\right)$. The value of $\Sigma(i, x, \eta, u)$ can change either because the trajectory of the system leaves the manifold $M_{i}$ and enters $M_{j}$, or because the discrete input $\eta$ changes. We will assume that as the dynamics of the system changes, the continuous state remains the same. In other words, the evolution of the continuous state $x$ will be continuous ${ }^{1}$. In general, the vector fields in $\mathcal{F}$ will be different, reflecting changes in the dynamics of the system. Also the dimensions of the manifolds in $\mathcal{M}$ might be different.

\section{Control strategy}

In this paper we study stabilization of SCD's. The control task is to stabilize a submanifold $E_{n}$ in a particular dynamic regime, $E_{n} \subseteq M_{n}$. Depending on the application, it might be necessary to achieve asymptotic stability or maybe only convergence of the trajectories of the system to $E_{n}$. In both cases, the control task is complicated by the fact that it is not known in advance what manifolds the dynamical system will traverse. In particular, it is possible that the system switches autonomously between different manifolds. It is also clear that switching might be unpredictable due to external disturbances.

Let $\Omega=(\Xi, \mathcal{M}, \mathcal{U}, \Gamma, \mathcal{F}, \Sigma)$ be a SCD. A natural way to control $\Omega$ is to design a controller for each of the dynamic regimes. Therefore, for each manifold $M_{i} \in \mathcal{M}$ we design a controller $g_{i}$ :

$$
g_{i}: M_{i} \rightarrow \mathcal{U}
$$

The evolution of the discrete state also depends on the discrete input $\eta$. We must therefore also design a discrete controller:

$$
\mathcal{S}: \Xi \times \cup_{i \in \Xi} M_{i} \rightarrow \Gamma
$$

which at each state $(i, x) \in \Xi \times \cup_{i \in \Xi M_{i}}$ selects a discrete input $\eta \in \Gamma$. Let $D(i, x)=\left\{\Sigma\left(i, x, \eta, g_{i}(x)\right) \mid \eta \in \Gamma\right\}$, the

\footnotetext{
${ }^{1}$ Most of the results in the paper can be generalized to systems with jumps.
}

set of all discrete states reachable from the current combined (discrete and continuous) state. We can force the system to switch to one of the discrete states that are reachable by choosing an appropriate discrete input. Without loss of generality we can assume that $\Gamma=\Xi$ and that whenever $j \in D(i, x), \Sigma\left(i, x, j, g_{i}(x)\right)=j$. For this reason, $\mathcal{S}$ will be also called a switching function. The collection of controllers $\mathcal{G}=\left\{g_{i}\right\}_{i \in \Xi}$ and the switching function (discrete controller) $\mathcal{S}$ form a switching controller, $(\mathcal{G}, \mathcal{S})$.

\section{Stability of switching controllers}

In the interest of space, the results in this section are stated without proofs. The complete exposition can be found in [15].

Since we are interested in stabilizing submanifolds (possibly unbounded), the conventional Lyapunov theory has to be appropriately extended for our setting (see for example [16]).

Take a $\operatorname{SCD} \Omega=(\Xi, \mathcal{M}, \mathcal{U}, \Gamma, \mathcal{F}, \Sigma)$ and a switching controller $(\mathcal{G}, S)$. Let the controller $g_{n}$ stabilize the manifold $E_{n}$ on $M_{n}$. Assume we can construct a Lyapunov function $V_{n}$ for $g_{n}$. The following proposition gives sufficient conditions for $E_{n}$ to be globally attractive:

Proposition 4.1 Let the switching scheme $S$ satisfy the following conditions:

(1.1) There exists $L>0$ such that $\mathcal{S}(n, x)=n$ for every $x \in$ $B\left(E_{n}, L\right) \cap M_{n}$ (a ball of radius $L$ around $E_{n}$, see [17]).

(1.2) For any trajectory $x(t)$, there exists $a \Delta>0$ and an infinite sequence $\left\{t_{i}\right\}$ whose elements satisfy:

(a) for every $t \in\left[t_{i}, t_{i}+\Delta\right], \mathcal{S}(\eta(t), x(t))=n$;

(b) $V\left(t_{i}+\Delta\right) \geq V\left(t_{i+1}\right)$.

Then the submanifold $E_{n}$ is globally attractive.

While Proposition 4.1 gives sufficient conditions for convergence of the system trajectories to $E_{n}$, these conditions are difficult to check and therefore not suitable for controller design. It is particularly difficult to check the condition (1.2), By introducing hierarchy among dynamic regimes (continuous controllers), we can obtain conditions that are easier to apply in the design process. The hierarchy will be formally defined through a partial order.

Definition 4.2 A binary relation on a set $A$ that is reflexive, transitive and antisymmetric is called $a$ partial order. We will denote it $b y \preceq$. If $a \preceq b$ and $a \neq b$, we also write $a \prec b$. If there exists an element $s \in A$ such that $s \preceq x$ for every $x \in A, s$ is called the smallest element. When it exists, the smallest element is unique.

Now let $\mathcal{M}=\left\{M_{i}\right\}_{i \in \Xi}$ be the collection of manifolds for a $\operatorname{SCD} \Omega$. The switching scheme $\mathcal{S}$ defines a relation $\sigma(\Xi)$, if 
we put $\sigma(i, j)$ when it is possible to switch from the manifold $M_{i}$ (controller $g_{i}$ ) to the manifold $M_{j}$ (controller $g_{j}$ ). More formally:

$$
\sigma(\Xi)=\left\{(i, j) \mid \exists x \in M_{i} \text { s.t. } \mathcal{S}(i, x)=j\right\}
$$

Proposition 4.3 Let $\sigma^{\text {Trans }}$ be the transitive closure of $\sigma$ and assume a partial order $\preceq$ within $\sigma^{\text {Trans }}(\Xi)$ that has $n$ for the smallest element. Let each controller $g_{i}$ asymptotically stabilize a manifold $E_{i}$ and assume we can find a Lyapunov function $V_{i}$ for $g_{i}$. Let the switching scheme $S$ satisfy the following conditions:

(3.1) For each $i$, there exists $L_{i}>0$ such that $S(i, x) \prec i$ for every $x \in B\left(E_{i}, L_{i}\right) \cap M_{i}$ (we require $S(n, x)=n$ ).

(3.2) There exists $\Delta>0$, such that for any $T$ at which the system switches from $g_{i}$ to $g_{j}, j \prec i, S(\eta(t), x(t)) \prec i$ for each $t \in[T, T+\Delta]$.

(3.3) If the system switches from $g_{i}$ to some $g_{j}, i \prec j$, at time $t_{\mathrm{off}}$ and after that switches again to $g_{i}$ at time $t_{\mathrm{on}}$ and if $S(\eta(t), x(t)) \nprec i$ for all $t \in\left[t_{\mathrm{off}}, t_{\mathrm{on}}\right]$, then $V_{i}\left(t_{\text {off }}\right) \geq V_{i}\left(t_{\text {on }}\right)$.

Then the submanifold $E_{n}$ is globally attractive.

\section{Design of switching controllers}

Proposition 4.3 can be used to design stable switching controllers. Design of the discrete controller will be guided by choosing a partial order on $\Xi$ (hierarchy among $M_{i}$ 's). The partial order can not be chosen arbitrarily, part of it is usually dictated by the physics of the problem. The continuous controllers on each $M_{i}$ must be then designed so that they are consistent with the chosen partial order. In particular, we need to satisfy the conditions (3.2) and (3.3) of the Proposition 4.3. We outline three techniques that can be used to satisfy these conditions.

\subsection{Hysteresis}

Hysteresis can be used to enforce the condition (3.2). Suppose we want to switch from $M_{i}$ to $M_{j}, j \prec i$. If $f_{j}\left(x, g_{j}(x)\right)$ in Eq. (1) is bounded for all $x \in H \subset M_{j}$, where $H$ contains $E_{i}$ and also the region in which the system switches from $g_{i}$ to $g_{j}$, then the condition (3.2) will be automatically satisfied if:

$$
\inf _{\{x \mid \mathcal{S}(i, x)=j\}} d(x,\{y \mid \mathcal{S}(j, y) \npreceq j\})>d_{0}
$$

where $d_{0}>0$ is some constant. In other words, after the switch from $M_{i}$ to $M_{j}$ we are some (fixed) finite distance from any point $y$ in $H$ where the system would switch to a discrete state which is not lower in the partial order. Because of the bounded rate of change of the continuous state, this implies that such a switch can only occur after some finite time interval. Hysteresis in the switching rule is also useful to eliminate chattering and increase the robustness of the system.

\subsection{Mixing of the controllers}

It is difficult to directly design controllers that would satisfy the condition (3.3). An alternative is to combine several controllers. More precisely, suppose we would like to allow switches from $M_{i}$ to $M_{j}, j \prec i$. To satisfy the condition (3.3), we need to have a controller $g_{i}$ that is able to decrease the Lyapunov function $V_{j}$. Controllers $g_{i}$ and $g_{j}$ stabilize $E_{i}$ and $E_{j}$, respectively. We also know that the controller $g_{j}$ decreases the Lyapunov function $V_{j}$. If $E_{j} \subset E_{i}$, we can construct a new controller, $\hat{g}_{i}$ that brings the system to $E_{i}$ and then moves the state along $E_{i}$ towards $E_{j}$. Chose some neighborhood $H$ of $E_{i}$ and define $\hat{g}_{i}$ to be:

$$
\hat{g}_{i}(x)= \begin{cases}g_{i}(x) & \text { if } x \in M_{i} \backslash H \\ g_{i}(x)+\varepsilon g_{j}(x) & \text { if } x \in M_{i} \cap H\end{cases}
$$

where $\varepsilon$ is a small constant. Now we can modify the switching scheme so that we do not switch from $g_{i}$ to $g_{j}$ until $V_{j}$ has sufficiently decreased. This will guarantee that the condition (3.3) is satisfied. To verify that $\hat{g}_{i}$ actually decreases $V_{j}$ while the system evolves on $B\left(E_{i}, L_{i}\right)$, we can use linearization around $E_{i}$.

\subsection{Dealing with autonomous switches}

Mixing of the controllers can be used to enforce the condition (3.3) when the switches between two discrete states $i$ and $j$ are under our control. But there will be situations when the system switches autonomously. In this case, it is helpful to decouple the autonomous switches from the switches between controllers with different equilibrium sets. Assume the system can switch autonomously from the discrete state $i$ to $j$, but the controllers $g_{i}$ and $g_{j}$ have different equilibrium sets. We can try to design a controller $g_{j}^{i}$ on $M_{j}$ that has the same equilibrium set and the same Lyapunov function as the controller $g_{i}$ on $M_{i}$. Then instead of switching from $g_{i}$ to $g_{j}$, we switch from $g_{i}$ to $g_{j}^{i}$. Since the controllers have the same Lyapunov function, the condition (3.3) is automatically satisfied. To switch to the controller $g_{j}$ (and enforce the convergence to a different equilibrium set), we then use mixing of the controllers $g_{j}^{i}$ and $g_{j}$, as described above.

\section{Example: Hilare robot}

We study control of a mobile robot of a Hilare type. A schematic of such a robot is shown on Fig. 1. The robot has two independently actuated wheels (the inputs are the torques $u_{1}$ and $u_{2}$ ). Since the dynamics of the robot change as the wheels switch between rolling and sliding, this is an example of a SCD. We wish to make the robot drive along a prescribed line in the plane with a constant forward velocity $v_{0}>0$ (such a line is a relative equilibrium). Referring to Fig. 1, the control task will be to stabilize the robot to the line $y=0$ and the velocity $v_{x}=v_{0}$.

The dynamics of the system changes depending on whether the wheels are rolling or sliding. We have four different regimes: (a) both wheels are rolling; (b) both wheels are sliding; (c) wheel 1 is rolling and wheel 2 is sliding; (d) 


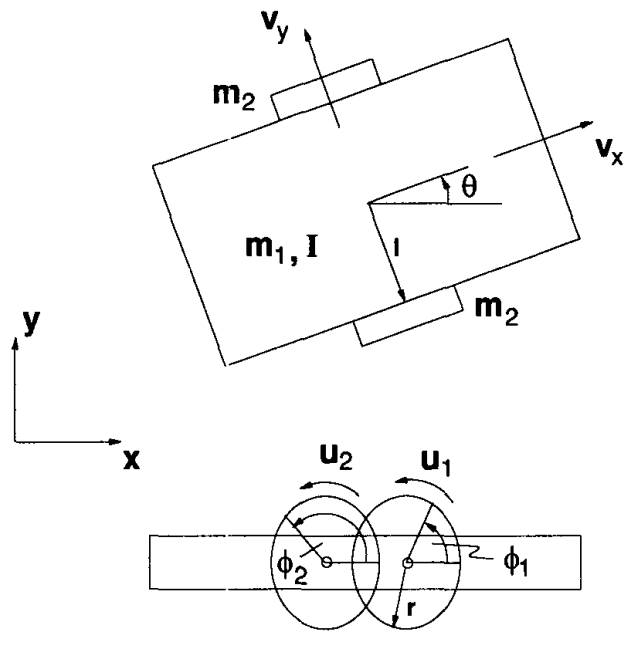

Figure 1: A top view and a side view of the Hilare robot

wheel 1 is sliding and wheel 2 is rolling. We denote these regimes by $R R, S S, R S$ and $S R$, respectively. In general, the Hilare robot can be described with 5 configuration variables: position of the center of mass ( $x$ and $y$ ) and orientation of the main body $(\theta)$, and the angles of rotation of both wheels $\left(\phi_{1}\right.$ and $\left.\phi_{2}\right)$. The phase space is therefore in general of dimension 10. If any of the wheels is rolling, additional relations among the state variables must hold and the dimension of the state space decreases.

Dynamics of the robot are given by the Euler-Poincare equations [18]:

$$
\begin{array}{rlrl}
\dot{w}_{1} & =\frac{F_{x}^{1} r+u_{1}}{I_{w}} & & \dot{v}_{x}=\omega v_{y}+\frac{F_{x}^{1}+F_{x}^{2}}{m_{b}+2 m_{w}} \\
\dot{w}_{2} & =\frac{F_{x}^{2} r+u_{2}}{I_{w}} & \dot{v}_{y} & =-\omega v_{x}+\frac{F_{y}^{1}+F_{y}^{2}}{m_{b}+2 m_{w}} \\
\dot{\omega} & =l \frac{F_{x}^{1}-F_{x}^{2}}{I_{b}+2 I_{v}} &
\end{array}
$$

The variables $w_{1}$ and $w_{2}$ are the rotational velocities of the wheels, $\omega$ is the rotational velocity of the body of the robot, $v_{x}$ is the forward velocity and $v_{y}$ the lateral velocity. In the equations, $m_{b}$ is the mass of the body of the robot, $m_{w}$ the mass of a wheel, $I_{b}$ is the moment of inertia of the body, and $I_{w}$ and $I_{v}$ are the moments of inertia of a wheel around its axis of rotation and around the vertical axis through its center of mass, respectively.

The configuration (group) variables can be obtained from the velocities by integrating the equation:

$$
\dot{g}=g \xi
$$

where $g \in S E(2) \times S^{1} \times S^{1}$ stands for the group variables and $\xi$ for the body-velocity (Lie algebra) variables [18]. In this paper, we are only interested in controlling the configuration variables $y$ and $\theta$, the rest of the variables are controlled at the velocity level. The dimension of the system is thus 7 . The forces $F^{1}$ and $F^{2}$ are the forces between the ground and the two wheels, expressed in the body-fixed frame. The indexes $x$ and $y$ stand for the forward and lateral directions. The rest of the symbols are explained in Fig. 1.
If the wheel 1 is rolling, we have two constraints:

$$
\begin{aligned}
v_{x}+l \omega+r w_{1} & =0 \\
v_{y} & =0
\end{aligned}
$$

Similarly, when the wheel 2 is rolling, the constraints are:

$$
\begin{aligned}
v_{x}-l \omega+r w_{2} & =0 \\
v_{y} & =0
\end{aligned}
$$

When the wheel $i$ is rolling, the force $F^{i}$ prevents slippage of the wheel and can be eliminated from Eq. (6) using Eq. (8) or Eq. (9). Since the rolling constraint results in two constraint equations, the dimension of the system drops by 2 whenever a wheel starts rolling. However, when both wheels are rolling, (8) and (9) only constitute 3 independent constraints. Therefore, when both wheels are rolling, the dimension of the system is 4 , including the configuration variables $y$ and $\theta$.

When the wheels are sliding, the reaction forces are frictional forces. The force $F^{i}$ when the wheel $i$ is sliding is:

$$
F^{i}=-\mu_{d} \frac{v_{r}^{i}}{\left\|v_{r}^{i}\right\|}\left(\frac{m_{b}}{2}+m_{w}\right) g
$$

where $\mu_{d}$ is the coefficient of (dynamic) friction, $g$ is the gravity constant and $v_{r}$ is the relative velocity between the point on the wheel which is in contact with the ground and the ground.

\subsection{Controller design}

The control task is to stabilize the robot to the relative equilibrium $E_{R R}=\left\{y=0, v_{x}=v_{0}\right\}$, a subset in the regime $R R$. The control strategy will be to first make the wheels roll and then stabilize the robot to the desired relative equilibrium. Note that once the wheels roll, we can not guarantee that no slippage will occur, the convergence to the desired set must be attained through switching.

Switches between rolling and sliding are autonomous (the discrete inputs have no effect on the switches). To deal with these autonomous switches we can use the method described in Section 5.3. This is possible since the equations for $w_{1}$ and $w_{2}$ in (6) can be feedback linearized regardless whether $F$ is a constraint force or a friction force. Let the linearizing controllers obtained by substituting the appropriate expressions for the force $F$ in different regimes be $g_{S S}, g_{S R}, g_{R S}$, and $g_{R R}^{1}$ (the last superscript indicates that we will need additional controllers in the regime $R R$ ). Using these controllers, we can drive the wheels to a constant value $-\frac{v_{0}}{r}$ (the nominal driving velocity). It can be shown [15] that these controllers drive the system to the regime $R R$ and asymptotically stabilize the set $E_{R R}^{1}=\left\{w_{1}=w_{2}=\right.$ $\left.-\frac{v_{0}}{r}, v_{x}=v_{0}, v_{y}=\omega=0\right\}$.

The next step is to design a controller in the regime $R R$ that stabilizes the robot to the desired equilibrium set. Consider the following two outputs:

$$
\begin{aligned}
& h_{1}=y+L \sin \theta \\
& h_{2}=v_{x}
\end{aligned}
$$


where $L$ is an arbitrary positive constant. Physically, $h_{1}$ corresponds to the $y$ coordinate of a point displaced by $L$ along the $x$ axis of the body-fixed reference frame, and $h_{2}$ to the forward velocity of the vehicle. Using these two outputs, we can design a controller that input-output linearizes the system [19]. Let this controller be $g_{R R}^{3}$. It can be shown that the zero dynamics of the system $(y)$ is asymptotically stable. The equilibrium set for the system is thus $h_{1}=v_{0}, h_{2}=\dot{h}_{2}=y=0$. Note that this equilibrium set contains values $\theta=k \pi$ for any $k \in \mathbb{Z}$. To guarantee that the system converges to $\theta=0$ it is thus necessary to design an additional controller, $g_{R R}^{2}$. We proceed similarly as before. We select the outputs we wish to control:

$$
\begin{aligned}
& h_{1}=\theta \\
& h_{2}=v_{x}
\end{aligned}
$$

and obtain the controller $g_{R R}^{2}$ by input-output linearization.

In total, we have designed six controllers: controllers $g_{S S}$, $g_{S R}$ and $g_{R S}$ in regimes $S S, S R$ and $R S$, respectively, and three controllers, $g_{R R}^{1}, g_{R R}^{2}$ and $g_{R R}^{3}$ in the regime $R R$. In order to use the model described in Section 2, we replace the manifold $M_{R R}$ with the manifolds $M_{R R}^{1}, M_{R R}^{2}$ and $M_{R R}^{3}$ (all equal to $M_{R R}$ ). The final partial order between the controllers is shown in Fig. 2: the partial order between the controllers $g_{S S}, g_{S R}, g_{R S}$ and $g_{R R}^{1}$ is induced by the physics of the problem, while the rest of the partial order was designed.

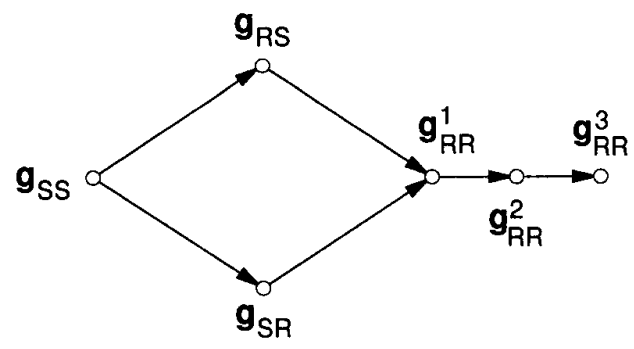

Figure 2: Partial order between the controllers.

The switching scheme (discrete controller) for the system in the regime $R R$ is:

$$
S(\eta, x)= \begin{cases}3 & \eta=3 \&|\theta|<\frac{\pi}{2} \\ & \&\left\|F_{c}^{1}\right\|<F_{g} \&\left\|F_{c}^{2}\right\|<F_{g} \\ 3 & |\theta|<\lambda \frac{\pi}{2} \&\left|v_{x}\right|<v_{0} \\ & \&\left\|F_{c}^{1}\right\|<\lambda F_{g} \&\left\|F_{c}^{2}\right\|<\lambda F_{g} \\ 2 & \eta=2 \&\left\|F_{c}^{1}\right\|<F_{g} \&\left\|F_{c}^{2}\right\|<F_{g} \\ 2 & \left\|F_{c}^{1}\right\|<\lambda F_{g} \&\left\|F_{c}^{2}\right\|<\lambda F_{g} \\ 1 & \eta=1 \&\left\|F_{c}^{1}\right\|<F_{g} \&\left\|F_{c}^{2}\right\|<F_{g}\end{cases}
$$

where $0<\lambda<1$ and $F_{g}=\mu_{d}\left(\frac{m_{b}}{2}+m_{w}\right) g$. The factor $\lambda$ in conditions (2) and (4) achieves hysteresis in the switches. The requirement $\theta<\frac{\pi}{2}$ in conditions (1) and (2) guarantees that the controller $g_{R R}^{3}$ stabilizes the system to the value $\theta=$ 0 (as opposed to $\theta=\pi$ ). Finally, the requirement $\left|v_{x}\right|<$ $2 v_{0}$ in condition (2) guarantees that $\dot{V}_{3}$ is negative when the system is controlled by $g_{R R}^{3}$. From $g_{R R}^{1}$, the system might switch to the regimes $S S, S R$, and $R S$ if any of the wheels starts sliding.

We refer the reader to [15] for the proof that the above controllers and the switching scheme satisfy all the conditions of Proposition 4.3 and therefore make the system converge to $E_{R R}$.

\subsection{Simulation results}

Figure 3 shows snapshots of a simulation run. The figures are shown at time intervals equal to $0.44 s$. The whole sequence spans $8 s$. The numbers in the figures represent the value of the switching function (controllers that were active): $3,2,1,-1,-2,-3$ correspond to $g_{R R}^{3}, g_{R R}^{2}$ and $g_{R R}^{1}$, $g_{R S}, g_{S R}$, and $g_{S S}$, respectively. The initial velocities of the wheels $\left(w_{1}\right.$ and $\left.w_{2}\right)$ were set to 0 while the velocities of the body of the vehicle were set to $(\dot{x}, \dot{y}, \dot{\theta})=(2,2,15)$. These initial velocities correspond to large initial relative velocities, giving rise initially to a period of sliding and a large excursion in the $y$ direction. During the sliding phase the robot also makes a full turn around its center. Both wheels stop sliding at time $1.57 \mathrm{~s}$ and the system switches to the controller $g_{R R}^{3}$. Two switches between $g_{R R}^{3}$ and $g_{R R}^{2}$ follow: at $1.75 \mathrm{~s}$ and $3.25 \mathrm{~s}$. The corresponding switches from $g_{R R}^{2}$ to $g_{R R}^{3}$ occur at $3.13 s$ and $4.46 s$. Some of the switches are not visible in Fig. 3 since they occur too fast. From time $4.46 \mathrm{~s}$ on, the controller $g_{R R}^{3}$ remains active and it finally stabilizes the robot to $E_{R R}$.

\section{Conclusion}

We investigated the problem of stabilizing systems with changing dynamics (SCD's). Such systems form a subclass of hybrid systems. A natural control strategy for stabilization of a SCD is to design a switching controller consisting of continuous controllers for each dynamic regime and a discrete controller that switches between them. We derived sufficient conditions for stability of a switching controller. Using the concept of partial order to introduce the hierarchy among continuous controllers, we have shown that the sufficient conditions can be reformulated in a way that naturally leads to a design methodology. We described three strategies that further simplify design of switching controllers: hysteresis in the switching rules, mixing of controllers in the same dynamic regime and decoupling of autonomous switches from the switches in the control objective. These techniques were applied to stabilization of a mobile robot of Hilare type whose wheels are allowed to slip. The example demonstrated that the proposed methodology naturally leads to a modularity in the design process.

An interesting problem that remains to be solved is how to stabilize a periodic orbit that passes through several discrete states (example is walking). It would be also worthwhile exploring whether the partial order is an inherent feature of every stable switching controller (necessary conditions). Finally, an open question is what classes of SCD's can be stabilized by purely state-dependent controllers and in which cases memory variables are essential for stabilization. 


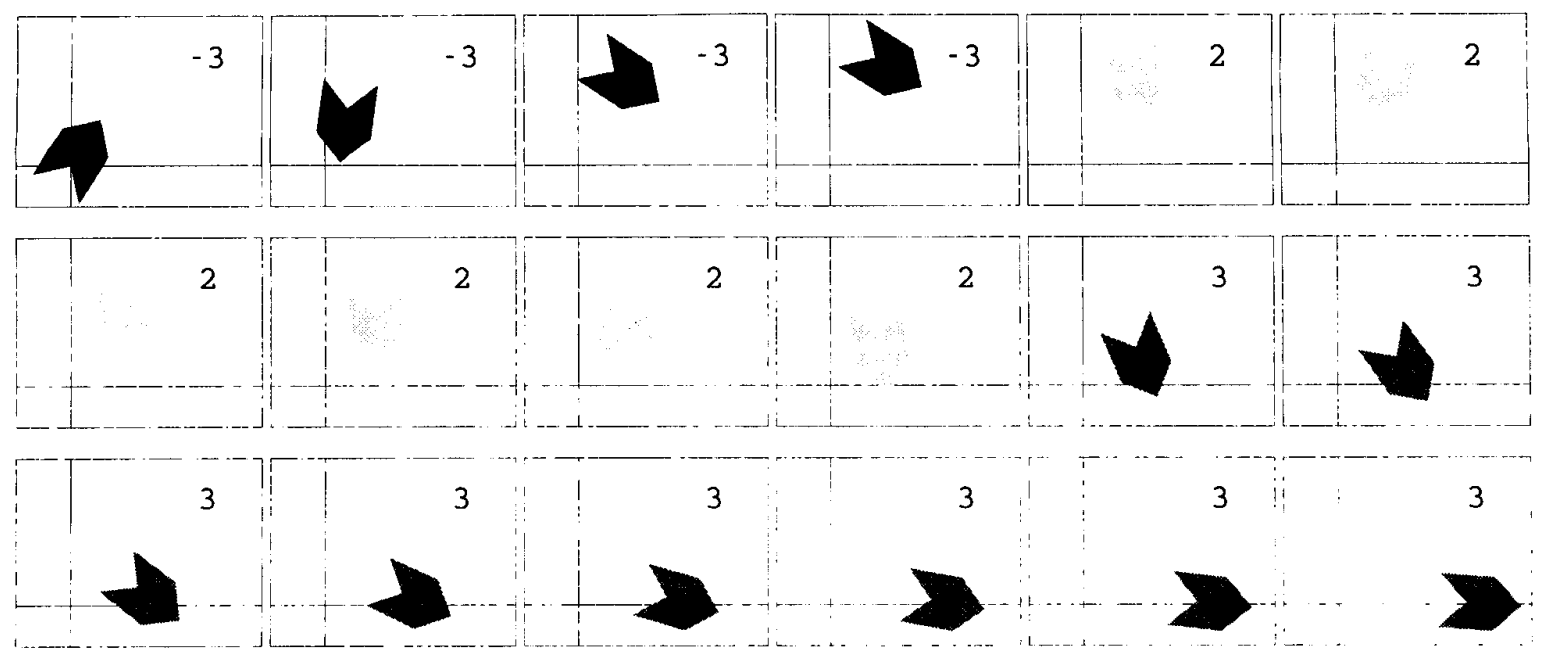

Figure 3: Snapshots of the motion of the robot.

\section{Acknowledgment}

The funding for this work has been provided by the NSF grant CSE-9704702 and the AFOSR grant F49620-96-10471 .

\section{References}

[1] E. D. Sontag, "Nonlinear regulation: The piecewise linear approach," IEEE Transactions on Automatic Control, vol. AC-26, pp. 346-358, 1981.

[2] A. Puri, Theory of hybrid systems and discrete event systems. $\mathrm{PhD}$ thesis, U. C. Berkeley, 1995.

[3] J. Lygeros, D. N. Godbole, and S. S. Sastry, "A game theoretic approach to hybrid system design," in LNCS 1066, pp. 1-12, Springer-Verlag, 1996.

[4] J. O. Moody and P. J. Antsaklis, "Supervisory control of petri nets with uncontrollable/unobservable transitions," in Proceedings of the 35th IEEE Conference on Decision and Control, (Kobe, Japan), 1996.

[5] I. Kolmanovsky and H. N. McClamroch, "Hybrid feedback laws for a class of cascade nonlinear control systems," IEEE Trans. on Automatic Control, vol. 41, no. 9, pp. 1271-1282, 1996.

[6] E. Sontag and H. J. Sussmann, "Nonsmooth controllyapunov functions," in Proceedings of the 34th IEEE Conference on Decision and Control, (New Orleans, LA), 1995.

[7] L. Hou and A. N. Michel, "Stability analysis of a general class of hybrid dynamical systems," in Proceedings of the 1997 ACC, (Albuquerque, NM), pp. 2805-2809, 1997.

[8] P. Peleties and R. DeCarlo, "Asymptotic stability of $\mathrm{m}$-switched systems using Lyapunov-like functions," in American Control Conf., (Boston), pp. 1679-1684, 1991.

[9] G. A. Lafferriere, "Discontinuous stabilizing feedback using partially defined Lyapunov functions," in Proceedings of the 33rd IEEE Conference on Decision and Control, (Orlando, FL), 1994.
[10] M. S. Branicky, "Stability of switched and hybrid systems," in Proceedings of the 33rd IEEE Conference on Decision and Control, (Lake Buena Vista, FL), pp. 3498-3503, 1994.

[11] J. Malmborg, B. M. Bernhardsson, and K. J. Åström, "A stabilizing switching scheme for multi-controller systems," in 13th IFAC World Congress, (San Francisco, CA), 1996.

[12] S. Pettersson and B. Lennartson, "Stability and robustness for hybrid systems," in Proceedings of the 35th IEEE Conference on Decision and Control, (Kobe, Japan), 1996.

[13] M. Johansson and A. Rantzer, "On the computation of piecewise quadratic Lyapunov functions," in Proceedings of the 36th IEEE Conference on Decision and Control, (San Diego, CA), 1997.

[14] R. R. Burridge, A. A. Rizzi, and D. E. Koditschek, "Sequential composition of dynamically dexterous robot behaviors.” Preprint, 1996.

[15] M. Žefran and J. W. Burdick, "Stabilization of systems with changing dynamics," IEEE Transactions on $\mathrm{Au}$ tomatic Control, 1998. Submitted.

[16] A. Vannelli and M. Vidyasagar, "Theory of partial stability theorems, converse theorems, and maximal Lyapunov functions," in Proc. Annu. Southeast Symp. Syst. Theory, (Piscataway, NJ), pp. 16-20, 1980.

[17] W. Hahn, Stability of motion. Springer-Verlag, 1967.

[18] J. E. Marsden and T. S. Ratiu, Introduction to mechanics and symmetry. Springer-Verlag, 1994.

[19] N. Sarkar, X. Yun, and V. Kumar, "Control of mechanical systems with rolling constraints: Applications to dynamic control of mobile robots," The International Journal of Robotics Research, vol. 13, no. 1, pp. 55-69, 1994. 